\title{
Discours
}

Revue de linguistique, psycholinguistique et

informatique. A journal of linguistics, psycholinguistics and computational linguistics

$17 \mid 2015$

Varia

\section{Procédures référentielles indexicales, relations rhétoriques et structuration du discours}

\section{Francis Cornish}

\section{OpenEdition}

Journals

Édition électronique

URL : http://journals.openedition.org/discours/9098

DOI : $10.4000 /$ discours. 9098

ISSN : 1963-1723

Éditeur :

Laboratoire LATTICE, Presses universitaires de Caen

\section{Référence électronique}

Francis Cornish, «Procédures référentielles indexicales, relations rhétoriques et structuration du discours », Discours [En ligne], 17 | 2015, mis en ligne le 22 décembre 2015, consulté le 19 avril 2019. URL : http://journals.openedition.org/discours/9098 ; DOI : 10.4000/discours.9098

\section{(c) $(1)(9)$}

Discours est mis à disposition selon les termes de la licence Creative Commons Attribution - Pas d'Utilisation Commerciale - Pas de Modification 4.0 International. 

Revue de linguistique, psycholinguistique et informatique

\section{Procédures référentielles indexicales, relations rhétoriques et structuration du discours}

Francis Cornish

CLLE-ERSS UMR 5263

Université de Toulouse - Jean Jaurès

Francis Cornish, «Procédures référentielles indexicales, relations rhétoriques et structuration du discours», Discours [En ligne], 17 | 2015, mis en ligne le 22 décembre 2015.

URL: http://discours.revues.org/9098

Titre du numéro: Varia

Coordination: Anne Le Draoulec et Josette Rebeyrolle 



\title{
Procédures référentielles indexicales, relations rhétoriques et structuration du discours ${ }^{1}$
}

\author{
Francis Cornish
}

CLLE-ERSS UMR 5263

Université de Toulouse - Jean Jaurès

Cet article défend l'idée que ce n'est pas le marqueur linguistique (pronom de $3^{e}$ personne, etc.) qui remplit de lui-même la fonction indexicale, qu'il s'agisse de deixis, d'« anadeixis » ou d'anaphore, mais la procédure référentielle indexicale choisie par le locuteur / le scripteur en tant que telle - procédure dont ce marqueur, au sein de la prédication hôte, est la réalisation.

À cet égard, on cherchera à distinguer, à l'instar d'Ehlich (1982), les procédures indexicales que sont la deixis, l'«anadeixis» et l'anaphore, et à préciser leurs fonctions discursives respectives. Chacune de ces procédures indexicales est spécialisée dans la réalisation de telle ou telle fonction de structuration et d'intégration du discours qui peut être associé à un texte quelconque, par le biais d'un contexte approprié. L'anaphore, elle, a partie liée avec les relations rhétoriques qui, de façon semblable, structurent le discours. Elle sert même à mettre en œuvre la relation rhétorique (ou de cohérence) reliant deux unités réunissant les conditions sémantiques et pragmatiques qui justifieraient l'application à celles-ci de la relation en question.

Mots clés : procédures référentielles indexicales, anaphore, deixis, anadeixis, texte, discours, contexte, marqueurs indexicaux, relations rhétoriques, structure aspectuelle et temporelle

This article argues that it is not the indexical expression in and of itself (whether a $3 r d$ person pronoun, etc.) which fulfils the indexical referring function, whether it is deixis, "anadeixis" or anaphora; but rather the indexical procedure chosen by the speaker/writer as such. It is this procedure which is realised by the indexical in question, within the host predication.

In this respect, we seek to distinguish, following Ehlich (1982), the indexical procedures corresponding to deixis, anadeixis and anaphora, and to make precise their respective discourse functions. Each of these procedures is specialised in realising various types of integrative structuring of the discourse which can be associated with a given text, via an appropriate context. As for anaphora, this procedure is closely bound up with the rhetorical or coherence relations which similarly structure a discourse. It even serves to implement the rhetorical relation connecting two units satisfying the semantic and pragmatic conditions which justify the application to them of the relation at issue.

Keywords: indexical referring procedures, anaphora, deixis, anadeixis, text, discourse, context, indexical expressions, rhetorical relations, aspectual and temporal structure

1. Cet article est la version remaniée et étendue d'une communication présentée lors du symposium de l'axe S'caladis «Sens dessus dessous: niveaux et domaines de réalisation du sens», tenu à l'université de Toulouse - Le Mirail du II au I2 avril 20I3. Le titre de cette communication était le suivant: «Référence indexicale et structuration du discours: incidences du choix de "procédure indexicale", de la/des relation(s) de cohérence invoquée(s), et de l'aspectualité». Je tiens à remercier pour leur relecture de versions antérieures de ce texte, Michel Aurnague et Co Vet ainsi que, pour ce qui concerne la version soumise à Discours, les deux relecteurs anonymes de la revue. 


\section{Introduction}

Dans la littérature sur la référence indexicale (l'anaphore surtout), la préoccupation centrale tend à se porter prioritairement sur la résolution des anaphoriques comme contribution à la représentation des états de choses désignés : il s'agit souvent dans ce cadre d'une approche vériconditionnelle de tels marqueurs. Or, bien que cet aspect soit important, il n'épuise pas toute la question. Il y a également la dimension interpersonnelle (interlocutive et intersubjective) du domaine, ainsi que celle de la contribution à la structuration du discours associé à un texte avec un contexte.

Dans tout renvoi indexical, ce n'est pas le marqueur linguistique - que ce soit un pronom de $3^{\mathrm{e}}$ personne, un pronom zéro, un pronom démonstratif, un syntagme nominal (SN) défini ou possessif, un $\mathrm{SN}$ démonstratif, etc. - qui remplit de lui-même cette fonction, mais la procédure référentielle indexicale ${ }^{2}$ choisie par le locuteur/le scripteur en tant que telle. Le marqueur sélectionné, conjointement avec la prédication hôte, n'est que le moyen rendu disponible par la langue pour réaliser cette procédure. Cette relation entre la langue-système et son emploi en contexte se reflète dans le fait bien connu que les mêmes formes (ici, indexicales) peuvent être employées dans des fonctions différentes, et que la même fonction pourra être remplie par des formes (indexicales encore) diverses. Cette mise au point a des ramifications importantes, comme on va le voir.

En outre, nous verrons que certains exemples textuels anglais comprenant des SN démonstratifs ne se comportent pas selon l'hypothèse de Gundel et al. (1993), en fonction de leur «Échelle du donné». Cette hypothèse exploite l'une ou l'autre des maximes de Quantité de Grice (1975) pour rendre compte de certains emplois «non canoniques» des marqueurs représentés sur cette échelle; et nous soutiendrons que l'emploi de ces marqueurs peut tout aussi bien s'analyser selon la typologie des procédures indexicales mobilisées dans tel ou tel contexte discursif par le locuteur. Sera examiné également le rôle, dans la structuration des textes, des prédications hôtes réalisant l'anaphore canonique ou un sous-type quelconque d'anadeixis, en étroite imbrication avec certaines relations rhétoriques. Nous évoquerons également l'incidence de certains phénomènes aspecto-temporels sur le fonctionnement des procédures indexicales et la mise en place des relations rhétoriques.

On commencera par mettre en place quelques préliminaires analytiques (section 2) avant d'aborder le propos central de l'article. La section 3 sera consacrée à la démonstration de la distinction de principe qu'il conviendrait de faire entre les marqueurs indexicaux, d'une part, et les différentes procédures indexicales qui se réalisent grâce à eux, d'autre part. Dans la littérature sur ce domaine, on a tendance à confondre les deux plans, en assimilant la procédure adoptée au marqueur lui-même. Il sera surtout question de la procédure appelée

2. Donc, la fonction discursive employée par le locuteur, le processus même de référenciation. 
«anadeixis». Nous aborderons aussi la question de l'étroite imbrication de la référence anaphorique et anadéictique inter-propositionnelle et de la mise en place des relations de cohérence (ou relations rhétoriques) afin d'intégrer l'unité «hôte» avec son unité «antécédente», créant ainsi une nouvelle unité de discours plus englobante (sous-section 4.I.). Pour terminer, on traitera brièvement en 4.2 de l'incidence de l'aspectualité sur le fonctionnement anaphorique et anadéictique, et sur l'intégration discursive plus généralement.

\section{Préliminaires analytiques: la triple distinction texte/contexte/discours, et les procédures indexicalo-discursives de deixis, d'anaphore et d'anadeixis}

Pour commencer, esquissons certaines distinctions terminologiques et conceptuelles, qui nous seront nécessaires pour la suite.

\subsection{La triple distinction entre texte, contexte et discours}

\begin{tabular}{|c|c|c|}
\hline Texte & Contexte & Discours \\
\hline $\begin{array}{l}\text { La suite connexe de signes } \\
\text { verbaux et de signaux non ver- } \\
\text { baux en fonction de laquelle } \\
\text { le discours est coconstruit par } \\
\text { les participants dans l'acte de } \\
\text { communication. }\end{array}$ & $\begin{array}{l}\text { Le contexte est assujetti à } \\
\text { un processus continu de } \\
\text { construction et de révision } \\
\text { au fur et à mesure que } \\
\text { le discours se déroule. } \\
\text { Le contexte comprend } \\
\text { les aspects suivants: le } \\
\text { domaine de référence d'un } \\
\text { texte donné, le cotexte, le } \\
\text { discours déjà construit en } \\
\text { amont, le genre d'événement } \\
\text { langagier en déroulement, le } \\
\text { cadre socioculturel supposé } \\
\text { par le texte, les relations } \\
\text { interlocutives existant entre } \\
\text { les interactants à chaque } \\
\text { moment de l'échange, et } \\
\text { la situation d'énonciation } \\
\text { particulière à l'œuvre. C'est } \\
\text { à travers l'invocation d'un } \\
\text { contexte approprié que } \\
\text { l'auditeur ou le lecteur } \\
\text { peut convertir en discours la } \\
\text { séquence connexe d'indices } \\
\text { textuels qu'est le texte. }\end{array}$ & $\begin{array}{l}\text { Le produit de la séquence } \\
\text { hiérarchisée et contex- } \\
\text { tuellement située d'actes } \\
\text { énonciatifs, indexicaux, pro- } \\
\text { positionnels et illocutoires } \\
\text { effectués dans la poursuite } \\
\text { d'un but communicatif } \\
\text { quelconque, et intégrés dans } \\
\text { un contexte donné. }\end{array}$ \\
\hline
\end{tabular}

Tableau 1. Rôles dans une séquence d'actes d'énonciation du texte, du contexte et du discours (tableau 1 dans Cornish, 2010a: 112 - partiellement révisé) 
Dans la littérature, il est fréquent qu'on confonde les termes (et partant, les notions) de texte et de discours; ou bien qu'on les considère comme quasi équivalents. Pour nous, le texte (au sens «massif» du terme, donc «du texte»: voir la définition proposée dans la colonne de gauche du tableau I) est la trace perceptible laissée par au moins un acte d'énonciation réalisé soit verbalement, soit non verbalement. En tant que tel, il comprend les traits paralinguistiques de ces actes, de même que des signaux non verbaux sémiotiquement pertinents (voir Clark, 1996: chap. 6). Le texte dans cette conception est essentiellement linéaire, à la différence du discours qui, lui, correspond à l'interprétation «en ligne», continue et sujette à révision, du texte, conjointement à un contexte approprié; ce contexte, à l'évidence, doit inclure les intentions communicatives du locuteur, telles qu'elles sont inférées par l'allocutaire (voir la colonne de droite du tableau i).

Le discours, lui, se crée à la fois à partir du texte et du contexte. Le discours dans cette acception, structuré en plans hiérarchiques selon la fonction de ses constituants essentiels, sera stocké ultérieurement dans la mémoire à long terme. En revanche, la trace textuelle de la communication disparaitra de la mémoire à court terme une fois que le discours sera construit puis restructuré.

S'agissant maintenant du contexte, sept aspects en sont décrits dans la colonne du milieu du tableau I, dont le plus important est sans doute la situation d'énonciation, qui opère comme point d'ancrage par défaut pour le discours à construire. Or, le contexte, qui est, comme le discours, mentalement représenté par les participants, n'est pas disponible en tant que tel dès avant la création du discours: il se (re-)construit tout au long de la communication, tout comme le discours lui-même. Car le discours en est non seulement tributaire, mais il le modifie dès lors qu'il se construit en temps réel ${ }^{3}$.

Or, exploitant cette distinction tripartite, mon hypothèse est qu'il existe, dans le fonctionnement de la référence indexicale, une interaction complexe entre les dimensions de texte et de discours, médiatisée par le contexte. Ce que Cornish (1999) appelle «le déclencheur d'antécédent» assure la catégorisation du référent de l'anaphorique. Un déclencheur d'antécédent peut être un énoncé, un fragment d'énoncé, l'objet d'une perception ou un geste sémiotiquement pertinent - des instances de texte, donc. Mais le référent lui-même et sa caractérisation sont déterminés en fonction de toute une gamme de facteurs d'ordre discursif: ce qui avait été prédiqué ou inféré de cette entité en amont de la reprise, la nature de la relation rhétorique invoquée afin d'intégrer les deux unités de discours à l'œuvre, et le caractère particulier de la prédication hôte.

Une fois le référent évoqué via un déclencheur d'antécédent donné, les interlocuteurs en formeront une représentation mentale. Cette représentation accumulera par la suite un certain nombre de propriétés, de relations, etc., telles qu'elles seront prédiquées ou inférées en fonction du texte subséquent en liaison avec un contexte approprié. Par la suite, l'occurrence d'un anaphorique (relevant

3. Voir aussi à ce sujet Auer (2009) et Cornish (2009a). 
donc du texte) conjointement à sa prédication hôte dans son ensemble, permettra à l'allocutaire ou au lecteur d'accéder à cette représentation telle qu'elle aura évolué au moment de la reprise. C'est là une illustration de la célèbre citation d'Héraclite, à savoir que «l'on ne met jamais le pied dans la même rivière par deux fois».

En matière d'anaphore, il n'y a de ce fait aucune relation intratextuelle directe posée entre déclencheur d'antécédent et anaphorique: voir Cornish (2orob) pour une discussion plus ample. Ainsi, contrairement à la conception textualiste classique de l'anaphore de discours, l'évocation directe et explicite du référent repris dans le cotexte précédent ou subséquent (dans le cas de la cataphore) ne fournit ni une condition suffisante, ni nécessaire, pour son existence. Pour l'utilisateur d'une langue naturelle, il n'y a pas de processus d'«appariement» entre deux expressions distinctes - l'antécédent textuel et l'anaphorique -, indépendamment de leurs environnements sémantico-pragmatiques respectifs, comme dans la conception traditionnelle. L'anaphore en tant que processus interprétatif n'est donc pas un phénomème purement cotextuel, impliquant la mise en relation de segments du cotexte (voir aussi Apothéloz et Reichler-Béguelin, 1999, parmi bien d'autres articles).

De ce fait, nous sommes en désaccord sur ce point avec la conception et la démarche essentiellement textualistes de Bordet (20II), parmi tant d'autres caractérisations récentes et plus anciennes. Voir Kehler (2008) pour des arguments convaincants, plus généralement contre ce qu'il appelle «le paradigme $\mathrm{SMASH}^{4}$ » adopté par les tenants d'approches privilégiant surtout les aspects formels de l'interprétation des pronoms de $3^{\mathrm{e}}$ personne, en particulier.

\subsection{Deixis, anaphore, anadeixis, et les propriétés indexicales distinctives de divers types d'expressions liées au contexte}

La deixis et l'anaphore sont toutes deux des procédures référentielles indexicales, que l'utilisateur exploite dans la construction, la modification et l'accession aux modèles de discours qui sont en gestation à tout moment de la communication. Chacun des participants construira un tel modèle, sorte de représentation mentale de l'événement communicatif ainsi que de son contenu, au fur et à mesure que celle-ci se déroule. Deixis et anaphore servent à gérer la coordination de l'attention des participants.

La fonction principale de la deixis est de guider l'allocutaire dans la construction mentale d'un nouvel objet de discours, par défaut à partir de la situation d'énonciation - dont le «point zéro» (l'«origo» selon Bühler, 2009 [1934]) est l'activité verbale et non verbale en déroulement du locuteur -, et ce conjointement avec l'allocutaire, devrait-on ajouter ${ }^{5}$. Elle assure ainsi l'ancrage du discours construit par la suite à travers la réalisation d'un texte dans un contexte pertinent (voir Langacker, 2002a) : la

4. Acronyme parlant fondé sur l'appellation «Search, Match and Select using Heuristics» (Kehler, 2008: 95).

5. Voir aussi Barbéris (2008: 200 et 203) et Fricke (2003) pour une discussion fort pertinente à cet égard, ainsi que Kibrik (2OII : 503). Plus récemment, Stukenbrock (2014: 76) le souligne aussi, en ce qui concerne la «Deixis am Phantasma» de Bühler (2009 [1934]). 
deixis crée ipso facto un contexte nouveau à chaque fois par l'établissement de valeurs particulières des paramètres contextuels de base ${ }^{6}$ pour l'événement communicatif; elle permet en même temps d'introduire la perspective subjective et la source du point de vue pour le discours en construction.

S'agissant maintenant de l'anaphore (de discours), on peut la concevoir comme constituant une instruction tacite en direction de l'allocutaire/du lecteur de garder tel quel le foyer d'attention déjà établi au moment de la parole - donc l'état du modèle de discours pré-existant. Ce sont le plus souvent les marqueurs indexicaux peu proéminents phonologiquement qui assurent cette fonction: de façon prototypique, les pronoms de $3^{\mathrm{e}}$ personne, les pronoms nuls, et les $\mathrm{SN}$ définis réduits.

Mais deixis et anaphore ne sont pas des procédures indexicales «symétriques» l'une de l'autre. Pour Lyons (1975) (voir aussi Bühler, 2009 [1934], Gerner, 2009, et d'autres), l'anaphore serait dérivée de la deixis et en dépendrait, la deixis étant une procédure référentielle plus élémentaire. Il y aurait entre ces deux procédures indexicales un continuum, avec un moyen terme. Ce «mi-chemin»- cette zone de chevauchement hybride - a été dénommé «anadeixis» par Ehlich (1982). Le préfixe grec ana- signifie «de nouveau» ou «en arrière», et dixis en grec saisit au sens strict le fait de pointer du doigt en situation pour montrer à l'interlocuteur le référent visé.

L'anadeixis représente une combinaison des procédures anaphorique et déictique dans des proportions différentes. Je relèverai trois sous-types majeurs d'anadeixis, comme suit:

- L'anadeixis «stricte» serait le renvoi ultérieur à un référent qui peut avoir été introduit antérieurement dans un discours, mais qui n'est plus - ou n'est pas encore - topical au moment de ce renvoi : le référent ainsi ciblé existe bel et bien dans le discours environnant, mais il est d'un accès relativement difficile, d'où l'implication de la dimension déictique.

- L'anadeixis de reconnaissance/de rappel impliquerait le pointage vers une entité - souvent un événement, parfois stéréotypique - présumée partagée au sein de la mémoire à long terme des participants. Ici, le référent ciblé existe également, indépendamment de cette référence indexicale; mais il est d'un accès encore plus difficile, étant localisé dans la mémoire à long terme des participants, d'où la primauté de la dimension déictique dans de tels renvois.

- Enfin, la deixis de discours correspondra au pointage cognitif vers une représentation discursive en mémoire de travail, et à la création par inférence à partir de celle-ci d'une entité discursive pour partie nouvelle, pour partie ancienne. Ici, le référent impliqué n'existe même pas en tant que tel en

6. L'espace déictique, le moment déictique, les rôles de locuteur actuel et d'allocutaire actuel dévolus à tel ou tel participant du discours, et la source du point de vue à l'œuvre. Voir aussi Hausendorf (2003) sur cette question. 
amont de l'acte de référence indexicale; la dimension déictique remplit de ce fait un rôle encore plus appuyé dans ce type de référence, d'où le nom de cette sous-procédure indexicale.

Ces trois cas de figure seront illustrés dans la prochaine section: exemples [2], [3] et $[\mathrm{I}]+[4]$, respectivement.

Penchons-nous à présent sur quelques représentants de la gamme des types de marqueurs indexicaux susceptibles de réaliser ces procédures, ainsi que leurs propriétés intrinsèques. Chaque marqueur, qu'il s'agisse de pronoms nuls, de pronoms de $3^{\mathrm{e}}$ personne manifestes, de noms propres réduits, de $\mathrm{SN}$ définis, démonstratifs ou possessifs, d'ellipses de divers types, possède un faisceau de propriétés indexicales, qui lui permet de réaliser des procédures discursivo-anaphoriques ou anadéictiques distinctes. Partant, chacun est sensible à différents types de contexte et de fonction discursifs.

Prenons pour commencer les SN démonstratifs marqués pour la distinction déictiquement pertinente «proximal» vs «distal» (les SN démonstratifs sans suffixe démonstratif du français moderne sont neutres à cet égard: voir la note 9 infra): les formes proximales ce $\mathrm{N}$-ci, celui-ci en français, et this (N) en anglais sont les membres marqués des paires de marqueurs concernés, les membres complémentaires distaux (ce $N$-là, celui-là et that (N)) étant non marqués. Employé en contexte, le nom tête des démonstratifs nominaux proximaux correspond normalement à de l'information non présupposée du référent à l'œuvre. Ce nom, accompagné ou non de modifieurs et/ou de compléments, sert plutôt à classifier le référent ciblé selon la perspective subjective adoptée sur le référent visé par le locuteur.

À la différence du composant lexical des SN démonstratifs, les noms têtes, le cas échéant avec leurs expansions possibles, au sein des SN définis ou possessifs ne représentent pas normalement des classifications provenant du locuteur: car la catégorie d'entités qu'ils dénotent est normalement présupposée de leur référent. En outre, les expressions définies réfèrent de façon «inclusive», alors que les démonstratifs non neutres le font de manière «exclusive»: leur emploi laisse entendre qu'il y a d'autres entités du même type qui ne sont pas incluses dans l'ensemble d'entités auquel ils renvoient. Cette propriété rend le premier sous-type de SN, à la différence du second, susceptible de réaliser l'anaphore (canonique): voir à cet égard le positionnement relatif de chaque sous-type de marqueur indexical dans l'échelle de la figure i ci-dessous.

Par hypothèse, l'emploi des formes démonstratives proximales plus généralement (ici, maintenant, ce $N$-ci, celui-ci en français et les expressions anglaises équivalentes bere, now, this (N)) exprime une implication personnelle, subjective, de la part du locuteur avec le référent; alors que celui des formes distales (là, alors, ce $N$-là, celui-là, et there, then, that (N)) suppose soit une dissociation personnelle de la part du locuteur par rapport au référent, soit un alignement entre locuteur et allocutaire à cet égard, où l'entité ciblée est présentée comme représentant de l'information déjà négociée, en termes interactionnels. 


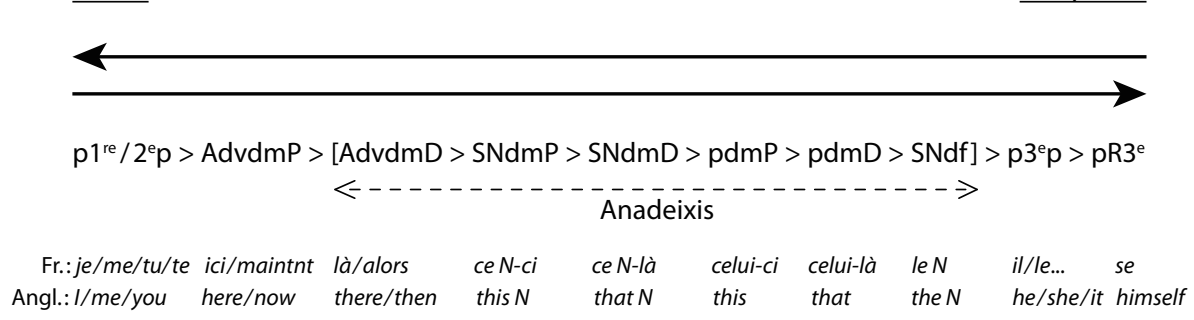

Figure 1. Échelle d'indexicalité encodée par certaines catégories d'expressions indexicales (figure 1 dans Cornish, 2010a: 116 - révisée)

L'emploi des pronoms de $3^{\mathrm{e}}$ personne référentiels inaccentués, pour sa part, porte la présupposition que le référent visé se trouve au centre de l'attention immédiate des interlocuteurs au moment de leur occurrence: il n'y a donc pas besoin d'initier une recherche cognitive coûteuse afin de le localiser. De tels marqueurs signalent la continuité de même que l'intégration du discours en déroulement.

La figure I range dix grandes catégories de marqueurs indexicaux sous forme d'une échelle de leurs propriétés indexicales intrinsèques ${ }^{7}$.

Cette échelle est fondée à partir du degré d'indexicalité inhérente que manifeste chaque catégorie indexicale individuelle. Ce qui motive le placement des marqueurs concernés à telle ou telle position sur l'échelle n'est pas uniquement la fréquence relative d'occurrences dans la réalisation de telle procédure indexicale, telle que manifestée dans les corpus, mais aussi, et surtout, les propriétés morpho-syntaxiques, sémantiques et discursivo-pragmatiques qui les rendent aptes à le faire.

Le pôle «Deixis» est occupé par les pronoms personnels de $\mathrm{I}^{\text {re }}$ et de $2^{\mathrm{e}}$ personne, souvent appelés «déictiques primaires», qui fonctionnent de manière «token-reflexive». À ce titre, ils ne s'emploient pas pour réaliser l'anaphore. Le pôle «Anaphore», quant à lui, est représenté par les pronoms réfléchis (clitiques en français) de $3^{\mathrm{e}}$ personne. Ces expressions, dans leur emploi prototypique, fonctionnent en termes de liage (une fonction anaphorique «stricte», donc), et, de plus, à l'intérieur d'une proposition: leur portée discursive est de ce fait très contrainte. Observons que la référence de

7. Les abréviations employées dans la figure $\mathrm{I}$ sont les suivantes: $« \mathrm{pr}^{\mathrm{re}} / 2^{\mathrm{e}} / 3^{\mathrm{e}} \mathrm{p} »=$ pronom de $\mathrm{I}^{\mathrm{re}} / 2^{\mathrm{e}} / 3^{\mathrm{e}}$ personne; «P»= proximal; «D»=distal; «dm»=démonstratif; «adv»=adverbe; «SN»=syntagme nominal; «p »=pronom; «df»=défini; «R»=réfléchi. À noter que l'ensemble des marqueurs anglais et français placés sous chaque catégorie indexicale dans l'échelle de la figure i n'a qu'une valeur illustrative: la relation clé entre chaque type de marqueur et la catégorie indexicale qu'il illustre est de nature intra-linguale uniquement. Leur relation ne devrait donc pas être lue «verticalement» (donc, dans une optique inter-linguale). Car il y a en effet des différences significatives par exemple, entre les SN définis en anglais et en français, de même qu'entre les pronoms réfléchis de $3^{\mathrm{e}}$ personne en français (qui sont clitiques) et en anglais (qui sont des expressions nominales, potentiellement argumentales, capables de ce fait de recevoir un accent de hauteur). 
ces deux types de marqueurs polaires se réalise quasi automatiquement, sans que le compreneur soit obligé de passer par un processus de résolution via le contexte.

Au milieu de l'échelle se trouve tout un empan marqué «Anadeixis». Il s'agit ici, pour la plupart, de types d'expressions à base démonstrative rangés entre les deux catégories polaires. Ces expressions démonstratives (adverbes, SN et pronoms) sont placées sur l'échelle en fonction de la distinction "proximale» (marquée) vs «distale» (non marquée) dont elles sont pourvues au plan morphologique - la variante marquée manifestant un degré plus élevé de déicticité que son homologue non marqué (voir Langacker, 2002b: 34; Lyons, 1975). Les adverbes démonstratifs (maintenant/alors, ici/là ${ }^{8}$ en français, et now/then, here/there en anglais) se trouvent à une position plus à gauche dans l'échelle que les SN lexicaux, tandis que ces SN lexicaux, eux, sont placés plus à gauche que les pronoms correspondants ${ }^{9}$. Les catégories à base démonstrative sont toutes ordonnées à gauche par rapport à la catégorie des $\mathrm{SN}$ définis sur cette échelle ${ }^{10}$. Ceux-ci sont situés à la frontière droite de l'empan «anadéictique» dans la figure I, car bien qu'ils soient susceptibles d'avoir des emplois déictiques, anadéictiques «stricts» ou anaphoriques, ils connaissent également des emplois non indexicaux ${ }^{11}$. Voir Cornish (2oroa et 20II) pour une discussion plus ample. Pour les pronoms anglais it, this et that et leur fonctionnement discursif, voir McCarthy (1994).

L'échelle reconnaît des positions relatives, et non «absolues», des catégories de marqueurs retenues. Il s'agit de ce que la langue-système rend disponible aux usagers pour référer indexicalement. Dans l'emploi en contexte, plusieurs facteurs peuvent intervenir pour moduler les valeurs systémiques que reflète cette échelle: la nature du nom dans le cas des marqueurs nominaux (SN), le cotexte environnant, la prosodie et d'autres facteurs contextuels encore dans d'autres cas.

8. Sur l'adverbe temporel maintenant, voir De Mulder et Vetters (2008), et pour ici et là, Kleiber (2008). Pour les premiers, l'adverbe proximal maintenant ne réalise que la deixis, et son homologue temporel distal, alors, fonctionne de préférence anaphoriquement (ou plutôt «anadéictiquement», devrait-on dire). Pour Kleiber, il en est de même pour la paire d'adverbes spatiaux ici (proximal) et là (distal).

9. Une exception à cette règle serait représentée par le $\mathrm{SN}$ démonstratif «nu» (sans suffixe démonstratif) ce $N$, s'il devait figurer dans cette échelle. Le déterminant démonstratif $c e$ n'est plus marqué pour la distinction «proximal»/ «distal», comme l'étaient les pronoms/déterminants cist et cil en ancien français. De ce fait, il n'exprime qu'une valeur déictique «faible» relativement au même SN étendu par l'un des suffixes démonstratifs, lorsqu'il est employé dans cette fonction (le marqueur ce $N$ peut avoir l'une ou l'autre des valeurs «proximale» ou «distale»); mais son emploi le plus fréquent à l'écrit ainsi qu'à l'oral de narration semblerait se limiter à l'expression de l'anadeixis, voire de l'anaphore pure. Cela reflète le fait qu'il est en passe de se grammaticaliser en article défini (voir De Mulder et Carlier, 2006). Pour toutes ces raisons, nous placerions ce marqueur dans l'échelle entre le pronom démonstratif distal celui-là et le SN défini.

10. Notons que ce placement relatif est en contradiction avec celui d'Ariel (2000) ainsi que de Gundel et al. (1993), dans leurs échelles d'«accessibilité» et de «statuts cognitifs» respectives.

11. En effet, ils sont à même de référer de façon autonome en fonction de leur seul contenu lexical, à condition que celui-ci soit suffisamment informatif pour que l'allocutaire ou le lecteur puisse identifier le référent de manière non équivoque. 


\section{Valeurs discursivo-pragmatiques en contexte des marqueurs indexicaux: les procédures indexicales vs l'«Échelle du donné » (Gundel et al., 1993)}

Pour bien saisir la pertinence de l'utilisation des procédures indexicales afin de prédire les valeurs discursivo-pragmatiques assumées en contexte par tel ou tel marqueur indexical, il sera utile de revoir certaines «échelles» de «statuts cognitifs» ou d'«accessibilité» des référents potentiels, qui ont été proposées pour un certain nombre de marqueurs. Des échelles de ce type, dont la «Givenness Hierarchy» (l'«Échelle du donné») de Gundel et al. (1993) est l'une des plus citées, conçoivent les marqueurs à l'œuvre comme ayant une relation directe avec le statut cognitif du référent qu'ils ciblent - relation «encodée», donc. Mais comme nous le verrons, il semble qu'on commette une sorte de confusion catégorielle ici : celle entre les propriétés internes des expressions retenues sous chaque position de l'échelle de statuts cognitifs selon l'étendue de leur portée cognitive ${ }^{12}$, déterminées donc par le système de la langue, d'une part, et leurs emplois possibles par un locuteur dans tel contexte d'énonciation, de l'autre. Or, les statuts cognitifs que tel ou tel marqueur peut cibler au niveau de ses référents potentiels sont flexibles, et non quasiment fixes $-\mathrm{du}$ moins pour leur statut cognitif «canonique»-, et c'est précisément en fonction de la procédure indexicale particulière qu'ils peuvent être amenés à réaliser en contexte (en emploi, donc), qu'on pourra déterminer ce statut.

La figure 2 représente l'«Échelle du donné» proposée par Gundel et al. (1993). Les six positions sont numérotées, pour plus de commodité.

En fait, seules les positions 4 («identifiable de façon unique») et 6 («identifiable quant au type d'entité dénotée»), statuts codés respectivement par les articles défini et indéfini, peuvent correspondre à un «statut» directement lié au faisceau de propriétés syntactico-sémantiques intrinsèques des marqueurs concernés. Les autres positions ( $1,2,3$ et 5 ) caractérisent toutes des effets de sens (ou «de référence», plutôt, ici), tributaires de l'emploi caractéristique des marqueurs concernés dans un contexte quelconque. Pour ce qui est, plus spécifiquement, de la position 5, il s'agit du statut «référentiel», caractéristique de l'emploi en première mention de this $N$ pour évoquer un référent revêtant une importance particulière pour le locuteur: voir en illustration l'exemple [I] plus bas. Or, ce statut traduit à l'évidence un type d'emploi particulier du marqueur. On le voit au sein de l'«Échelle du donné» elle-même, dans le fait que ce même type de marqueur se trouve également ailleurs (à la position 2, "activé»). L'exemple [I] fournit une illustration attestée orale (en anglais) de l'emploi du SN démonstratif proximal repéré à la position 5 de l'échelle.

12. Le statut le plus restrictif à cet égard est celui d'«en focus», qui se trouve au pôle gauche de l'«Échelle du donné» (figure 2 plus bas), et le statut le moins restrictif (ou le plus «ouvert») est représenté par le statut «identifiable selon le type d'entité dénotée» présent au pôle droit. 


\begin{tabular}{|c|c|c|c|c|c|}
\hline 1 & 2 & 3 & 4 & 5 & 6 \\
\hline en focus $>$ & activé & $>$ familier $>$ & $\begin{array}{l}\text { identifiable de } \\
\text { façon unique }\end{array}$ & $>$ référentiel & $\begin{array}{l}>\text { identifiable } \\
\text { quant au type } \\
\text { d'entité dénotée }\end{array}$ \\
\hline it & $\begin{array}{l}\text { that/this } \\
\text { this N }\end{array}$ & that $N$ & the $N$ & $\begin{array}{l}\text { this } N \\
\text { «indéfini» }\end{array}$ & $a N$ \\
\hline
\end{tabular}

Figure 2. La «Givenness Hierarchy» (Gundel et al., 1993 : 275)

[I] [...] and we were wal/, you know, walking out and we saw this thing and we're going "Have you paid and displayed ?" like this and we came back to the car, [rires] and there were this parking ticket slapped on the window screen [sic] and, I said to Tim, "where's that ticket?" [rires] I said "have you”, I said, "did you get”, I said "did you pay for the car?" he said "yeah”, I say "where's that ticket, it won't", "it's in my pocket" [rires] [...].

(Extrait de «elascı» : conversation libre, région du Lancashire, projet sur la «Phonologie de l'anglais contemporain» [PAC] en ligne à l'adresse suivante: http://www. projet-pac.net/images/samples-audio/elascrig.pdf. Ici la locutrice [SCI] raconte un incident où elle avait trouvé un procès-verbal sur le pare-brise de sa voiture)

J'analyserai le SN démonstratif proximal this parking ticket slapped on the window screen (sic) ("ce procès-verbal collé sur le pare-brise») en gras dans [I] comme réalisant la fonction «deixis de discours» (voir aussi l'exemple [4] plus bas). C'est à ce titre qu'il en vient à assumer le statut «référentiel», au sens de l'«Échelle du donné»; il n'y a rien de «catégorique» d'une telle valeur pour ce marqueur. Selon Prince (198I: 235), l'emploi de this déterminant illustré dans [I] sert à introduire un topique nouveau. Il est conceptuellement indéfini, et évoque toujours un référent spécifique. Ici, la référence est empreinte d'une forte charge de subjectivité, le propre des introductions au moyen de cette construction existentielle there $+b e+t h i s ~ N . .$. Il possède plusieurs propriétés qui le distinguent de l'emploi déictique «canonique» du démonstratif.

Selon nous, cependant, ce type d'expression indexicale possède un seul et unique faisceau de propriétés sémantico-pragmatiques intrinsèques; et la différence exprimée à travers son placement à des positions différentes dans l'échelle découle directement des procédures indexicales différentes qui sont mobilisées dans chaque cas de figure: la deixis de discours dans celui du statut «référentiel» (exemple [r]), et l'anadeixis «stricte» dans le cas de la valeur «activée» (voir l'exemple [2] ci-dessous).

Nous allons voir à présent d'autres statuts cognitifs qui peuvent correspondre à des emplois distincts des mêmes types de marqueurs: ici les $\mathrm{SN}$ démonstratifs proximaux et distaux this $N$ - en l'occurrence, these $N$ dans [2] - et that $N$, respectivement. D'abord, l'emploi anadéictique «strict» du SN proximal these children illustré dans l'exemple [2], correspond, comme prédit par l'«Échelle du donné», au statut «activé»: 
[2] Anadeixis «stricte»:

\section{The switched-on generation}

The debate about the watershed goes back and forth like a ping pong ball. The parents of the children who watch unmonitored TV are not likely to be writing to your page. As a teacher in inner-city areas for years, I was faced every morning with bleary-eyed seven-year-olds.

These children have no bedtime. There is no such thing as bath, teeth cleaning and a cosy story. The reality is that many have their own TV in their bedroom, and as long as they are quiet... Some even just fall asleep in the living room, and are carried off to bed when the parents eventually go.

The lives of these cbildren would shock complacent middle-class writers. How do we protect the thousands of children who regularly watch till Io or IIpm, born into families where parenting skills have never been acquired?

(Liz Bailey, Oldbury, West Midlands, lettre au magazine Radio Times, 4-Io août zooI, p. 7)

Le référent générique du SN démonstratif proximal these children ( $\mathrm{I}^{\mathrm{re}}$ occurrence) a déjà été mis en exergue dans le premier paragraphe de cette lettre: on peut donc considérer qu'il est topical dès avant ce renvoi, car les enfants dont il est question sont d'ailleurs le thème principal de ce texte. C'est le contexte ré-initialisateur représenté par la transition vers l'évocation de la raison de la situation décrite dans le premier paragraphe - selon l'auteur -, qui motive cet emploi «anadéictique strict». Ce nouveau topos est donc traduit formellement par l'ouverture d'un nouveau paragraphe, et la fonction «anadéictique stricte» de these children s'y conforme.

À noter que ni le pronom pluriel they, ni un SN défini parallèle (the children) n'auraient été naturels dans ce contexte, car ces types de marqueurs sont spécialisés dans l'expression de la continuité référentielle et thématique pure. Dans le cas du SN défini, cela représente au moins l'une de ses fonctions discursives possibles: voir les exemples [5]-[7] plus bas ${ }^{13}$. Le troisième paragraphe, qui porte sur la question des remèdes à la surfatigue des enfants le matin à l'école, sans toutefois les préciser, emploie d'ailleurs le même marqueur pour renvoyer au macrotopique de ce court texte. Notons en passant que, par hypothèse, en tant qu'enseignante des classes primaires dans des écoles au centre des grandes villes, l'auteur aurait choisi le membre marqué (proximal) du déterminant démonstratif plutôt que son homologue distal dans les deux cas, afin d'exprimer (tacitement) son empathie et sa solidarité avec les enfants dont il est question: voir la sous-section 2.2 sur ce point.

De même, l'emploi en «anadeixis de reconnaissance» du SN démonstratif that $N$ dans l'exemple [3], pourrait bien correspondre au statut «familier» indiqué sur l'«Échelle du donné» pour ce type de marqueur (position 3): voir that light, fresh

13. Voir Pu (20II : 99) sur les frontières entre unités de discours dans des textes anglais et chinois, tant oraux qu'écrits, et les effets que peut produire un déplacement d'épisode sur les types d'indexicaux employés pour reprendre des référents anciennement topicaux, comme c'est le cas dans l'exemple [2]. 
summer look which is perfect at bome or on boliday. Mais le point essentiel est que c'est à travers son emploi pour réaliser cette procédure qu'il a cette valeur, et non uniquement en fonction de ses propriétés intrinsèques, relevant du système de la langue:

[3] Anadeixis de reconnaissance:

Ioo \% Natural Leather upper with a slight wedge heel plus a cushioned foot bed for extra comfort and support. Also, an elastic and flexible ankle strap for a perfect fit every time! Wear any time and with any outfit for that light, fresh summer look which is perfect at bome or on boliday [...].

(Publicité: «Ladies Woven Sandals», supplément «Premier Offers Direct», Radio Times, 16-22 août 20I4, p. 4)

Ici, il y a un aspect déictique à la référence du SN démonstratif distal that light, fresh summer look which is perfect at home or on holiday, au sens où l'attention du lecteur est orientée vers une représentation présumée partagée en mémoire épisodique - pour les lecteurs féminins, en tout cas, à qui cette publicité s'adresse. C'est là la raison d'être de la suite d'épithètes pré-modificatrices valorisantes light, fresh summer et de la séquence which is perfect at home or on holiday, post-modificateur au sein du SN lui-même, qui agissent comme des indices de récupération mémorielle (une sorte d'«amorce») pour le lecteur.

En même temps, il y a une dimension anaphorique à ce renvoi, car l'emploi de ce SN présuppose l'existence préalable de la représentation partagée (le type de «look» des sandales d'été dont il est question) au sein de la mémoire à long terme du lecteur: autrement dit, que cette caractérisation lui sera familière. Il ne s'agit aucunement de construire une telle représentation mémorielle, effet que pourrait avoir un renvoi déictique canonique ou déictique de discours: voir l'exemple [4] pour ce dernier type. Cependant, cette référence est néanmoins plus nettement déictique qu'anaphorique, car il ne peut y avoir de présomption de la part du scripteur que l'attention de son lecteur soit déjà focalisée sur le référent visé dans ces exemples - même de façon périphérique, comme c'est le cas des renvois anadéictiques «stricts» (voir l'exemple [2]).

[4] Deixis de discours:

Paul Temple was one of the great fictional detectives from the heyday of that/?\#the genre and had a radio career that stretched from 1938 to 1968 [...].

(«Paul Temple and the Sullivan Mystery», Radio Times, 5-II août 2006, p. I24)

Cependant, dans le cas du sous-type de procédure indexicale qu'est la deixis de discours ${ }^{14}$, à l'évidence, ni le statut cognitif «activé» (comme en [2]) ni le statut «familier» (comme en [3]), retenus par l'«Échelle du donné», ne seraient pertinents pour caractériser le référent créé à partir du traitement du segment de discours

14. Voir l'emploi du SN démonstratif distal that genre dans l'exemple [4]. 
précédent, car son existence discursive est le fruit d'une inférence ${ }^{15}$ tirée au moment du traitement de ce marqueur, à partir de ce qui est prédiqué du personnage Paul Temple - à savoir, one of the great fictional detectives from the beyday of that genre -, en fonction des connaissances du monde du lecteur.

À ce titre, au moment où la prédication Paul Temple was one of the great fictional detectives from the heyday of... that genre est traitée par le lecteur, le fait que le personnage de Paul Temple relève du genre du roman policier des années I930 et après n'est ni en focus ni même accessible (ni «familier», comme prédit [à tort] par l'«Échelle du donné» - voir la figure 2). Comme nous venons de le dire, son existence discursive est le résultat d'une inférence tirée lors de l'interprétation de l'indexical: pour y parvenir, le prédicat complexe fictional detectives, qui permet de classifier le personnage de Paul Temple, doit être "démonté» afin d'accéder à la catégorie «fictional detective stories» (romans policiers), et partant au «genre» de littérature que ceux-ci représentent. D'où l'emploi du SN démonstratif distal that genre pour renvoyer à cet aspect du personnage évoqué. Voir, à cet égard, Webber (I99I : I26) et Diessel (1999).

Comme on l'a déjà dit au sujet du fonctionnement du SN démonstratif proximal en [I], étant donné qu'avec la deixis de discours c'est sur le discours environnant qu'opère l'allocutaire (ou le lecteur) afin de s'approprier le référent visé ${ }^{16}$, alors son statut cognitif serait plus proche de la position 5 («référentiel») sur l'«Échelle du donné», que de la position 3 («familier») que ce marqueur est censé encoder au plan systémique. Pourtant, ce statut se trouve deux positions plus bas (vers la droite) sur l'échelle par rapport à son statut «codé».

Voilà pour les SN démonstratifs. Pour les SN définis réalisant une fonction purement anaphorique, voir les exemples [5] et [6] (sous-section 4.I), ainsi que [7] (sous-section 4.2) plus bas.

Or, certes, l'«Échelle du donné» a une certaine flexibilité inhérente, car, en contexte, des occurrences de certains types de marqueurs peuvent bien cibler un référent dont le statut cognitif est plus restrictif (donc situé plus à gauche sur l'échelle) que celui qu'il est censé encoder «en langue». Il faut se rappeler que la hiérarchie a un caractère implicationnel: en effet, à toute position sur l'échelle, l'item lexical qui lui correspond est censé encoder ce statut tout en impliquant tous les statuts inférieurs, donc ceux qui se trouvent à sa droite sur l'échelle: car un marqueur se trouvant plus à gauche par rapport à un autre sur l'échelle aurait un caractère plus «fort», donc plus restrictif en termes cognitifs, qu'un autre situé à sa droite. Il est de ce fait logique qu'un statut plus restrictif puisse impliquer un ou des statut(s) qui l'est ou le sont moins.

Cependant, Gundel et al. (1993) mobilisent la première maxime de Quantité proposée par Grice (1975) dans le cadre de son «Principe de Coopération», qui est

15. À savoir, «le roman policier des années I930-I960 est un genre particulier de littérature».

16. En l'anticipant dans le cas du SN démonstratif proximal dans l'exemple [I]. 
celle-ci: «Faites en sorte que votre contribution soit aussi informative qu'il est nécessaire (pour les besoins de l'échange)». Cette maxime est exploitée afin de poser que le locuteur laisserait entendre par ce biais que l'emploi d'un marqueur plus «fort» («impliquant») n'est pas de mise. En anglais, par exemple, les locuteurs ont tendance à ne pas employer les pronoms démonstratifs pour reprendre des référents ayant le statut d'«en focus», statut qui est à gauche de celui d'«activé» que coderait ce type de marqueur sur l'«Échelle du donné». Pour les auteurs, la raison en serait que leur emploi n'exige que le statut d'«activé» (voir la position 2 de la figure 2); ainsi, les employer pour exprimer la valeur précédente sur l'échelle («en focus») ne reviendrait pas à «faire en sorte que la contribution [du locuteur] soit aussi informative qu'il est nécessaire». Dans ce cas, donc, une implicature serait créée selon laquelle le statut d'«en focus» n'est pas de mise.

La seconde maxime de Quantité est celle-ci: «Ne faites pas en sorte que votre contribution soit plus informative qu'il n'est nécessaire». En respectant cette maxime, l'emploi d'un type de marqueur plus «faible» (donc impliqué) situé à droite sur l'«Échelle du donné» par rapport à celui à sa gauche impliquerait conversationnellement que c'est le statut plus «fort» (impliquant) qui est bien de mise.

Les auteurs proposent en illustration les SN introduits par l'article défini en anglais. Par convention, ce type de marqueurs est censé coder le statut «identifiable de façon unique» («uniquely identifiable») à la position 4. Mais il peut impliquer en outre, grâce à l'invocation de la seconde maxime de Quantité de Grice (1975), le statut plus fort, plus restrictif donc, de «familier». L'exemple qu'en donnent les auteurs est celui d'un panneau qui, tout en informant le destinataire de l'existence d'un objet quelconque, en même temps la présuppose - comme dans Mind the step/gap! («Attention à la marche/à l'écart!») ou Beware of the dog! («Gare au chien!»).

Pourtant, l'emploi du SN démonstratif distal that genre dans l'exemple [4] suppose dans son contexte le statut plus «faible» de «référentiel», mais n'implique pas en même temps, précisément, ceux de «familier» et d'«identifiable de façon unique». Or, comme on l'a vu, le statut «référentiel» se trouve à deux positions plus à droite de la position que ce marqueur est censé encoder sur l'«Échelle du donné» (à savoir, «familier»). L'hypothèse de Gundel et al. (1993) ne prévoit pas du tout cette possibilité - pourtant avérée selon nombre d'exemples de nos corpus, dont l'exemple [4] est une illustration. Toutefois, c'est là une conséquence naturelle de l'exploitation de l'une ou l'autre des procédures référentielles indexicales étudiées ci-dessus.

Les conclusions qu'on pourra tirer de cette discussion sont de deux ordres: d'abord, on constate que c'est la nature de la procédure référentielle indexicale exploitée qui détermine ces différentes valeurs référentialo-discursives, et non l'invocation de l'une ou l'autre des maximes de Quantité de Grice par l'allocutaire ou le lecteur, en tant que telle. Et ensuite, on remarque qu'il est nécessaire, lorsqu'on veut spécifier les valeurs référentielles et discursivo-cognitives exprimées en contexte par tel ou tel marqueur indexical, de distinguer entre les types de marqueurs à l'œuvre, d'une part, et leur emploi en contexte par un locuteur, d'autre part. 
Deixis pure $>$ Deixis de discours $>$ Anadeixis de reconnaissance $>$ Anadeixis «stricte» $>$ Anaphore pure

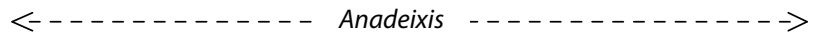

Figure 3. Échelle des procédures référentielles indexicales

(figure 2 dans Cornish, 2010a: 121)

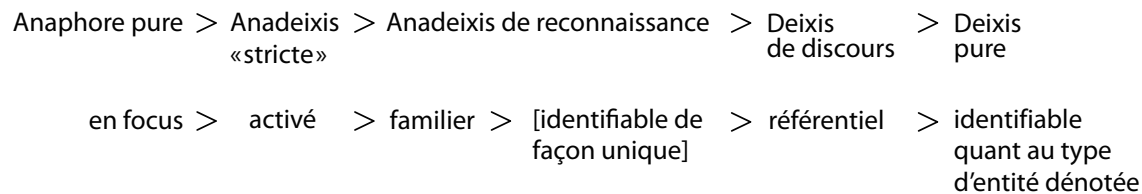

Figure 4. Superposition de la figure 3 inversée sur la figure 2

Pour terminer cette section, la figure 3 résume les diverses procédures indexicales évoquées précédemment, mais en incorporant également les deux procédures «polaires» de deixis canonique et d'anaphore canonique - à nouveau sous forme d'échelle. Il s'agit en fait de l'homologue «procédural» de la figure I, qui proposait de caractériser les propriétés indexicales d'une gamme de marqueurs indexicaux.

Si maintenant, nous inversons l'orientation de cette échelle, et la superposons à l'«Échelle du donné» de la figure 2, nous verrons que les deux échelles sont en corrélation presque parfaite (voir la figure 4).

Les deux positions au pôle droit de chaque échelle ont en commun le fait de reconnaître la fonction qui consiste à introduire un référent qui est nouveau pour le discours: la deixis pure en réorientant l'attention de l'allocutaire en fonction de l'acte d'énonciation en contexte, et le SN indéfini, prototypiquement, en amenant un nouvel objet de discours à l'existence discursive en fonction de la catégorisation de cette entité fournie via le prédicat lexical de ce SN.

La différence (majeure) entre les deux échelles est à l'évidence la présence dans l'«Échelle du donné» du statut d'«identifiable de façon unique», statut ciblé de façon prototypique, selon les auteurs, par les SN définis. Mais il n'y a aucune procédure indexicale dans l'Échelle des procédures indexicales (figure 3) qui pourrait y correspondre. Ce statut serait donc «orphelin» au niveau des positions disponibles sur l'Échelle des procédures. Car ce type d'expression peut être soit indexical, réalisant la deixis, l'anadeixis «stricte» ou l'anaphore, soit non indexical au sens où il fonctionne de façon autonome par rapport à son seul contenu lexical, là où celui-ci suffit pour cibler de façon non ambiguë un référent - donc sans que l'utilisateur soit obligé de passer par le contexte pour le faire ${ }^{17}$. Vue depuis l'Échelle des procédures indexicales, donc, cette situation est tout à fait prévisible.

17. C'est en réalité cet emploi qui est reconnu à la position 4 de l'«Échelle du donné». 


\section{Incidences du choix de la relation rhétorique invoquée et de l'aspectualité}

\subsection{Incidence de l'invocation de telle ou telle relation rhétorique (ou de cohérence)}

Le rôle joué par les marqueurs indexicaux dans la cohésion et la cohérence de textes multi-propositionnels est fondamental. Une condition sur l'isotopie nécessaire pour qu'un marqueur maintienne, en le reprenant, un référent saillant (ou même relativement peu saillant) évoqué au sein d'une unité discursive voisine, est l'existence d'au moins une relation de cohérence (ou relation rhétorique) permettant l'intégration des deux unités en présence en une unité d'ordre supérieur, englobante. Si aucune relation rhétorique ou de cohérence n'est disponible en contexte pour intégrer les deux unités, alors il est possible que le ou les marqueurs indexicaux dans la seconde unité soit/soient ininterprétable(s). En outre, dans des exemples textuels comme celui-ci,

\section{[5] Paulson offered treasury role}

President Bush nominated Henry Paulson, the chief executive of Goldman Sachs, as US treasury secretary in place of John Snow. The 6o-year-old investment banker is a China expert and keen environmentalist.

(The Guardian Weekly, 9-I5 juin 2006, p. 2; exemple [13] dans Cornish, 2010b : 226)

il est clair que la relation d'Élaboration d'entité ${ }^{18}$ qui pourra être invoquée afin d'intégrer les unités de discours macro qui correspondent aux deux phrases du texte, pourra seulement «élaborer» l'individu macrotopical introduit dans l'unité de discours constituant la phrase initiale, mentionné aussi dans le titre. Car ce texte porte évidemment sur Henry Paulson, et non sur John Snow: c'est Henry Paulson qui allait être à l'époque le nouveau secrétaire américain au Trésor. John Snow, lui, se retire, cédant sa place à Paulson. Snow n'est plus en poste, et le lecteur ne s'attend pas à ce que le reste du texte porte sur lui. Une prédication ne peut prédiquer quelque chose que d'un référent qui aura été auparavant singularisé comme susceptible de recevoir de l'information nouvelle: étant donné le caractère aspectuellement statif de même que prédicatif de cette seconde phrase, qui en tant qu'énoncé «catégorique» et non «thétique» suppose l'existence d'un argument référentiel auquel l'information qu'il véhicule puisse s'appliquer, elle sert à attribuer une nouvelle propriété à... Henry Paulson.

Or, c'est précisément à travers la coréférence entre les deux SN concernés dans ce court texte que cette «élaboration» peut s'effectuer. En réalité, la relation fortement préférée d'Élaboration d'entité sous-tendant l'intégration de ces deux unités de discours oblige la reprise en continuité par l'anaphorique de cette entité discursive. Si l'on s'efforçait d'interpréter le SN défini sujet de la seconde phrase dans l'exemple [5]

18. Voir Prévot, Vieu et Asher (2009) pour cette relation. 
comme coréférant à «John Snow», alors dans ce cas, il ne serait plus possible à l'unité de discours correspondant à celle-ci d'élaborer, indirectement du moins, celle que représente la première; car aucune des deux ne porte, en effet, sur l'entité «John Snow». C'est donc le référent mentionné en premier qui est la cible du SN sujet défini étendu de la seconde phrase (the 6o-year-old investment banker, SN qui est déjà une «élaboration» de cette entité) - même si ce SN pourrait, en principe du moins ${ }^{19}$, cibler le référent «John Snow».

Le point clé ici est que la relation anaphorique qui s'établit entre le marqueur défini the 6o-year-old investment banker et Henry Paulson, le référent macrotopical de ce court texte, fournit l'ancrage, le "pivot» nécessaire à la mise en place de la relation de cohérence qu'est l'Élaboration d'entité afin d'intégrer, de manière indirecte, les deux unités de discours en une unité de rang supérieur. C'est là un préalable à la compréhension du texte dans son ensemble - donc pour construire le discours qui pourra être associé à ce texte, voir le tableau I. Car pour «élaborer» une entité donnée, cette entité doit figurer à la fois dans la situation élaborée et dans l'élaborante.

Voici à présent un exemple textuel français, une brève de la rubrique des «faits divers» cette fois. Cet exemple permet de saisir une autre fonction remplie par l'anaphore dans l'intégration des unités de discours dans les textes: celle qui consiste à sélectionner l'unité à intégrer avec celle du segment hôte - unité qui peut ne pas être contiguë à celle-ci, au plan textuel.

[6] Trois policiers de la police aux frontières (PAF) de l'aéroport de Roissy ont été mis en examen, fin novembre, pour «homicide involontaire», par un juge d'instruction de Bobigny (Seine-Saint-Denis). En janvier, un Éthiopien âgé de 24 ans qui devait être reconduit, sous escorte, sur un vol vers l'Afrique du Sud, était décédé. Les fonctionnaires avaient été suspendus le 22 janvier.

(Le Monde, 13 décembre 2003, p. I2)

Ici, il y a quatre unités de discours (désormais abrégées UD), correspondant au plan textuel aux trois phrases complètes du texte en plus de la relative incorporée dans la deuxième (dans son $\mathrm{SN}$ sujet ${ }^{20}$ ). Nous allons les noter, dans l'ordre, UD ${ }^{0}$ à UD³. La phrase initiale, énoncé «thétique» ou "tout en focus», présente la situation à développer dans sa généralité: notons à cet égard le $\mathrm{SN}$ sujet indéfini ici. La situation présentée via la deuxième phrase n'est pas reliée explicitement à celle-ci;

19. En tout cas, pour les lecteurs qui n'auraient aucune connaissance préalable des deux individus en présence ici. De plus, il faut noter ici la pertinence de l'information posée en apposition sur Henry Paulson à la première ligne du texte, selon laquelle il était chief executive of Goldman Sachs: or, si le lecteur sait que Goldman Sachs est non seulement une «banque», mais de plus une «banque d'investissement», alors ce sera là le facteur décisif dans le choix parmi les deux référents en lice.

20. Cette relative serait par hypothèse appositive, malgré l'absence de virgules autour d'elle, et non déterminative, car elle pourrait être supprimée sans conséquences syntaxiques, sémantiques ni référentielles. Cependant, dans ce cas, son apport discursif serait sérieusement en défaut. À ce titre, elle pourrait bien correspondre à une unité de discours à part entière, à la différence des relatives déterminatives. De plus, elle évoque un événement (du moins en perspective). 
cependant, le cadratif temporel en janvier joint au temps plus-que-parfait du verbe de la principale (était décédé) décale la temporalité attendue de la nouvelle situation en faisant précéder le moment de cet événement de celui de la situation cadre - unité d'arrière-plan au niveau du discours, donc.

Par défaut, la situation évoquée par l'UD' (la deuxième unité de discours majeure du texte) assume une relation d'Explication avec celle-ci : le décès de l'Éthiopien ainsi évoqué correspondra par hypothèse au décès motivant la mise en examen des trois policiers de la $\mathrm{PAF}$, à savoir l'«homicide involontaire». Cela laisse entendre, bien entendu, que ces trois individus avaient fait partie de «l'escorte» conduisant l'Éthiopien en question sur le vol vers l'Afrique du Sud. Ces deux évocations permettent d'assurer l'isotopie nécessaire à une interprétation cohérente des deux UD concernées.

La situation évoquée par l'UD', à savoir la suspension des trois policiers dont il s'est agi dans l'UD ${ }^{0}$, est comprise comme étant intervenue à la suite de l'événement évoqué par les $\mathrm{UD}^{1+2}$. Mais l'UD ${ }^{3}$ 'est pas intégrée avec les $\mathrm{UD}^{1+2}$ en tant que telles, car ces dernières ne font aucune mention explicite, ni même implicite, des trois policiers. Observons que l'UD ${ }^{2}$ serait intégrée dans l'UD' en fonction de la relation de Circonstance. C'est donc en partie par le biais de la relation anaphorique exprimée via le SN défini les fonctionnaires et établie en vertu de la relation hyperonymique entre policiers et fonctionnaires, que l'UD ${ }^{3}$ est intégrée à l'UD ${ }^{0}$ (à laquelle seront déjà intégrées les $\left.\mathrm{UD}^{1+2}\right)$, qui est l'unité «cadre» de ce court texte. La relation soustendant la dernière intégration, celle entre l'UD ${ }^{0}$ et l'UD's serait celle de Résultat.

Il s'agit bien d'une relation d'ordre anaphorique et non anadéictique «strict» ici, car la structure de discours reliant l'UD ${ }^{0}$ et l'UD (l'unité «hôte») n'est pas interrompue comme dans le cas du texte de l'exemple [2] : l'unité composite $\mathrm{UD}^{\mathrm{I+2}}$ qui les sépare est à l'évidence dépendante par rapport à l'UD ${ }^{0}$. Elle ne constitue aucunement une unité autonome, à part entière, contribuant à l'avant-plan du discours. Il y a donc une continuité du discours ainsi créé, ce qui favorise évidemment l'anaphore. Comme nous l'avons vu, cela était impossible (ou très difficile) dans le cas des deux occurrences de SN démonstratifs remplissant la fonction d'anadeixis «stricte» dans l'exemple [2]. C'est sans doute le sous-genre des brèves qui fait préférer l'emploi du SN défini lexical par rapport au pronom dans l'exemple [6] ${ }^{21}$. La structure de discours associée à ce texte serait donc, schématiquement, celle-ci :

$\left[6^{\prime}\right] \quad\left[\left[\mathrm{UD}^{0}\left[{ }_{\mathrm{Expl}} \mathrm{UD}^{1}\left[{ }_{\text {Circ }} \mathrm{UD}^{2}\right]\right]\right]\left[_{\mathrm{Res}^{e s}} \mathrm{UD}^{3}\right]\right]$

21. À ce sujet, voir Corblin (2005) qui fait l'hypothèse, plus généralement, que, à la différence des interlocuteurs dans une conversation qui, eux, feraient partie d'une «communauté épistémique» restreinte, l'écrit serait un type de discours «à interlocuteur générique». C'est à ce titre que les reprises anaphoriques manifesteraient une plus grande variation de marqueurs (par exemple, l'emploi des SN définis à tête lexicale variée, des $\mathrm{SN}$ démonstratifs, etc.). Dans les articles de journaux, on trouve fréquemment ce genre de variation dans les reprises, les SN définis souvent étendus étant particulièrement favorisés (voir l'exemple anglais en [5]). C'est ainsi que le journaliste peut introduire de façon plus concise des informations nouvelles sur les individus et objets évoqués, stimulant l'intérêt de ses lecteurs et développant progressivement leur caractérisation. 
Voir Kehler et al. (2008) et Kehler (2008) ainsi que Cornish (2009b et c) pour une discussion plus ample sur les relations étroites qui existent entre le fonctionnement discursif de l'anaphore et les relations de cohérence.

\subsection{Incidence de l'aspectualité sur le fonctionnement de l'anaphore ou de l'anadeixis}

Les temps et aspects, l'Aktionsart ${ }^{22}$, la voix, les adverbiaux temporels, les relations temporelles et aspectuelles existant entre les propositions concernées, et plus généralement la structure événementielle de la prédication en jeu ${ }^{23}$, jouent un rôle non négligeable dans le fonctionnement de la référence indexicale et dans l'intégration des UD associées aux textes.

Dans l'exemple anglais en [5], la phrase initiale «cadre» renvoie à un événement spatio-temporellement situé : le temps du verbe (nominated), prédicat d'achèvement au niveau de l'aspect lexical, est le prétérit. Cependant, la seconde phrase n'est pas de nature à faire avancer la temporalité de cet événement (on n'est pas dans la narration), mais a un statut purement prédicatif: la copule be au temps présent simple introduit un prédicateur attribuant des propriétés. Cette configuration correspond parfaitement à la définition de la relation d'Élaboration d'entité, car le SN sujet dénote un individu, de plus, macrotopical du point de vue discursif: nous restons dans la même situation que celle évoquée par la phrase initiale - un événement qui recouvre l'état dénoté par la seconde phrase de ce texte. Voir aussi Dowty (I986) à cet égard.

Dans l'exemple [6] qu'on vient de voir, la dépendance pragmatico-discursive de la seconde phrase vis-à-vis de celle qui la précède, également une prédication d'événement spatio-temporellement situé, est surtout signalée par le temps plusque-parfait, qui, comme dans la phrase qui la suit, ne fait pas avancer la narration des événements mais opère un retour en arrière. De tels retours ont souvent une valeur explicative ou ont pour but d'effectuer un commentaire sur les faits présentés.

La troisième et dernière phrase («hôte») comporte également un verbe d'achèvement (suspendre) au plus-que-parfait, ainsi qu'à la voix passive. Ces facteurs contribuent à mettre en exergue ici l'état résultant de l'événement clé qui le précède : mais cette fois le temps de l'événement évoqué se situe après, et non avant, celui posé par la phrase précédente. C'est la relation rhétorique de Résultat invoquée ici grâce (en partie) à la configuration grammaticalo-lexicale qu'on vient de voir, qui détermine cette valeur. La cohérence d'ensemble de ce court texte est fondée sur le fait que l'état de choses évoqué par cette dernière phrase est impliqué déjà par la phrase cadre initiale, en fonction de notre connaissance du monde: «si des policiers sont mis en examen, alors il est probable qu'ils aient été suspendus de leurs fonctions au préalable».

22. L'aspect lexical des prédicateurs verbaux des deux propositions à relier: statuts d'état, d'activité, d'achèvement ou d'accomplissement.

23. Voir Rothstein (2004) pour une discussion éclairante sur cette dernière. 
Voici, pour terminer, un dernier exemple comportant cette fois un référent implicite, ciblé par un SN défini à fonction anaphorique:

[7] FAITS DIVERS

Un gendarme renversé

$\left[\mathrm{UD}_{\mathrm{EV} / \mathrm{FOND}}^{0}{ }^{24}\right.$ Un gendarme a été renversé et blessé au genou et à la cheville $\left[\mathrm{UD}_{\mathrm{LOC}}^{\mathrm{1}}\right.$ ${ }_{\text {TEMPS }}$ dans la nuit de samedi à dimanche, $\left[\mathrm{UD}_{\mathrm{LOC}}^{2}\right.$ à Saint-André-de-Cubzac (Gironde), [UD ${ }_{\mathrm{EV} / \mathrm{CIRC}}^{3}$ lors d'un contrôle routier]]]].

$\left[\mathrm{UD}_{\mathrm{ET} / \mathrm{FGGURE}}^{4}\right.$ L'automobiliste, [UD ${ }_{\mathrm{ET} / \mathrm{EL} \mathrm{LAB}-\mathrm{ENT}}$ toujours en fuite], est activement recherché]].

(20 Minutes, 5 mai 20I4, p. 6)

Dans le texte [7], également une brève de journal, nous avons une macro-unité discursive initiale présentant l'événement cadre du texte, introduit par le biais d'un énoncé thétique ou «tout en focus», le sujet étant un SN indéfini. Enchâssées dans cette unité se trouvent trois micro-unités localisantes, la première (UD') situant l'événement dans le temps par rapport à la date de parution de cette édition du journal, la seconde $\left(\mathrm{UD}^{2}\right)$ dans un lieu précis, et la troisième (UD³) évoquant les circonstances du fait. Elles fonctionnent toutes trois comme cadratifs. Du point de vue aspecto-temporel, l'énoncé cadre initial est à la voix passive (courte), le temps étant le passé composé où l'aspect accompli est en exergue (voir aussi Adam, 2000: IIO-III).

De par ces deux facteurs, c'est l'état résultant de cet événement qui est mis au premier plan. Il s'agit donc d'un événement ponctuel localisé. L'unité majeure suivante $\left(\mathrm{UD}^{4}\right)$ est présentée comme dépendante par rapport à l'unité cadre, de par la présence en position sujet d'un $\mathrm{SN}$ défini réduit non autonome référentiellement, donc dans une relation de dépendance référentielle, autrement dit, indexicale; mais aussi de par le fait que la phrase en question est une passive d'état (adjectivale), toujours «courte» comme la phrase passive de départ. Les deux unités impliquées par la seconde phrase du texte (UD' ${ }^{4}$ et $\mathrm{UD}^{5}$ ) manifestent une valeur inaccomplie du point de vue aspectuel, ce qui souligne la dépendance référentialo-discursive de cette unité composée par rapport à celle du départ.

Quant au marqueur indexical l'automobiliste (en gras ici), il reprend le référent implicite évoqué via le traitement du verbe au passif renverser (prédicat d'achèvement) dans l'UD ${ }^{0}$ et l'emploi du syntagme prépositionnel circonstanciel lors d'un contrôle routier dans l'UD's ${ }^{3}$ À ce titre, il permet à l'UD ${ }^{4}$ d'être intégrée à l'unité cadre au moyen de la relation Fond-figure (ou Arrière-plan). Cette inférence est clairement de type «connectif» plutôt que simplement «élaboratif», selon Zwaan

24. Les abréviations des notations en indice caractérisant les UD ici sont les suivantes: «LOC»=localisation spatiale; «LOC-TEMPS»= localisation temporelle; «EV»=événement; «ET»=état. Voir la procédure notationnelle adoptée par Smith (2003). Les notations suivant les barres obliques insérées après les indications de la structure événementielle des UD concernées représentent les relations rhétoriques à l'œuvre, c.-̀̀-d. FOND-FIGURE, CIRC(ONSTANCE), ÉLAB(ORATION)-(D’)ENT(ITÉ). 
et Rapp (2006: 735) 25. Si l'accident dont a été victime le gendarme a eu lieu «lors d'un contrôle routier», alors un véhicule quelconque a dû y être impliqué - par défaut une voiture, pilotée, encore par défaut, par un conducteur (donc un «automobiliste»). De par les connaissances du monde mobilisées par le lecteur, le gendarme en question aura participé au contrôle routier dont il est question.

Une autre inférence, de type causal cette fois, sera nécessairement tirée dans un deuxième temps: à savoir que c'est parce que le conducteur en question avait quelque chose à se reprocher aux yeux des policiers effectuant le contrôle routier, qu'il a cherché à les éviter, conduisant de ce fait dangereusement - ce qui, à son tour, aura eu comme conséquence le heurt du gendarme. La situation évoquée dans les deux UD majeures concernées est donc la même, le second des deux énoncés principaux renvoyant à un état de choses existant au moment du traitement de ce segment du texte, et non à un nouvel événement (voir Dowty, 1986).

\section{Conclusions}

La leçon la plus significative que l'on peut tirer de ce qui précède concerne l'importance qu'il y a à bien faire la distinction entre ce qui relève du système de la langue et ce qui appartient à l'emploi en contexte des ressources que ce système met à la disposition de l'usager. Dans notre cas, ce sont les différents types de marqueurs référentiels, chacun avec son faisceau particulier de propriétés morpho-syntaxiques, sémantiques et pragma-discursives, qui relèvent de la langue-système; et leur emploi en contexte est tributaire des procédures indexicales que sont la deixis, l'anadeixis et l'anaphore. Comme nous l'avons amplement vu ci-dessus, les mêmes types de marqueurs peuvent remplir des fonctions discursives distinctes, ce qui aboutit à l'attribution de statuts cognitifs différents aux référents concernés en contexte: il n'y a donc pas de quasi «bi-univocité» entre terme et fonction ou statut cognitif, comme les échelles du style de Gundel et al. (1993) pourraient le laisser supposer - bien que l'«Échelle du donné», il est vrai, permette un certain degré de flexibilité à cet égard (voir à ce sujet la discussion en section 3). En dernière analyse, la distinction entre les trois grands types de procédure indexicale permettrait de façon plus sûre de prévoir la valeur discursivo-pragmatique des marqueurs qui, conjointement à la prédication hôte dans son ensemble, les réalisent dans tel ou tel contexte d'énonciation.

L'anaphore joue un rôle important dans la mise en place des relations rhétoriques dont c'est également la fonction d'assurer l'intégration d'unités de discours attribuées à certaines unités textuelles: à savoir, celui d'agir comme pivot entre deux unités

25. Une inférence «élaborative» implique une activation de connaissances qui rehaussent la représentation mentale de la situation évoquée, mais dont on n'a pas besoin pour intégrer les deux assertions; alors qu'une inférence "connective» constituerait un moyen de relier deux assertions entre elles (Zwaan et Rapp, 2006: 735). 
discursives, assurant ainsi l'isotopie nécessaire à l'application de la relation rhétorique et créant de ce fait une unité discursive composite, supérieure. La prédication hôte dans son ensemble sert également à sélectionner parmi un sous-ensemble d'unités de discours voisines celle qui lui sera intégrée en fonction d'une relation anaphorique ou anadéictique, et partant, d'une relation rhétorique. L'unité textuelle réalisant l'unité de discours voisine choisie dans ce but ne sera pas nécessairement contiguë à la prédication hôte concernée.

Les facteurs aspecto-temporels jouent également un rôle important, non seulement en ce qui concerne l'attribution d'une relation indexicale (anaphorique ou anadéictique) entre deux unités textuelles, mais également dans le choix d'une relation rhétorique cohérente avec cette attribution, comme nous l'avons vu en 4.2. Voir Cornish (2009c: 162-166) pour une revue de ces facteurs, parmi d'autres indices heuristiques: à savoir, les relations temporelles et aspectuelles existant entre les propositions à l'œuvre - temps du verbe, adverbiaux temporels, l'Aktionsart des prédicateurs respectifs concernés, puis la structure événementielle des deux unités plus généralement. Le tableau I (Cornish, 2009c: I64) résume et synthétise ces facteurs. Pour les emplois des temps verbaux dans les textes, notamment les brèves journalistiques de faits divers, et la pertinence de leurs valeurs aspectuelles associées, voir Adam (2000) pour une étude éclairante.

Enfin, la distinction entre anadeixis et anaphore permet de restreindre le champ de cette dernière procédure: dans la littérature, ce terme est largement employé pour des phénomènes qui recouvrent également ce qui relève plus exactement de l'anadeixis - procédure qui, comme on l'a vu, ne possède pas les mêmes conditions d'emploi. Un exemple récent se trouve dans Bordet (201I : I2-19), où l'auteur présente comme «anaphoriques» les différents exemples attestés d'occurrences de this déterminant ou pronom analysé. Or, l'anaphore de discours proprement dite se cantonne à assurer la continuité référentielle entre certaines unités de discours, fournissant ainsi un indice important de leur intégration et de leur unité (voir Fox, 1987).

L'anadeixis «stricte», quant à elle, sert (parmi d'autres fonctions) à signaler la transition entre unités de discours mineures lorsque, par exemple, le référent qu'elle cible était topical dans l'unité qui vient de se terminer (voir l'exemple [2]); et l'une des fonctions discursives remplies par la deixis de discours est également de marquer la transition, cette fois entre unités majeures de discours. Elle le fait en synthétisant la contribution discursive de l'empan de texte qui précède, tout en annonçant le propos essentiel de l'unité de discours majeure qui est ainsi amorcée: voir à cet égard les exemples textuels anglais longs analysés dans ce sens dans Cornish (2008 et 20iI). Leur mode de fonctionnement discursif est tourné à la fois vers l'amont du discours, et vers l'aval.

L'anaphore et l'anadeixis peuvent donc remplir des fonctions particulières de signalement de la structuration du discours. 


\section{Références}

AdAM, J.-M. 2000. Le temps et les temps dans les textes. In J. Moeschler et M.-J. ReichleRBÉGUELIN (éd.), Référence temporelle et nominale: actes $d u 3^{e}$ cycle romand de sciences $d u$ langage, Cluny (15-20 avril 1996). Berne: P. Lang: I07-I2I.

Apothéloz, D. et Reichler-BÉGuelin, M.-J. 1999. Interpretations and Functions of Demonstrative NPs in Indirect Anaphora. Journal of Pragmatics 3I (3) : 363-397.

Ariel, M. 2000. The Development of Person Agreement Markers: From Pronouns to Higher Accessibility Markers. In M. Barlow et S. Kemmer (éd.), Usage-Based Models of Language. Stanford: CSLI Publications: 197-260.

Auer, P. 2009. Context and Contextualisation. In J. Verschueren et J.-O. Östman (éd.), Key Notions for Pragmatics. Amsterdam - Philadelphie: J. Benjamins : 86-IoI.

BARBÉRIs, J.-M. 2008. La deixis spatiale dans les descriptions d'itinéraires piétons : comment s'orienter dans l'espace de la ville? In M. Vuillaume (éd.), Ici et maintenant. Cahiers Chronos 20. Amsterdam - New York: Rodopi : 199-219.

BORDET, G. 20II. «This» comme marqueur privilégié du genre: le cas des résumés de thèses. Discours 9: I-27. En ligne à l'adresse suivante: http://discours.revues.org/8506.

BüHLER, K. 2009 [1934]. Théorie du langage: la fonction représentationnelle. D. SAMAIN (éd.). Banc d'essais. Marseille: Agone.

Clark, H. H. 1996. Using Language. Cambridge - New York: Cambridge University Press.

Corblin, F. 2005. Les chaînes de la conversation et les autres. In J.-M. Gouvard (éd.), De la langue au style. Lyon: Presses universitaires de Lyon: 233-254.

Cornish, F. 1999. Anaphora, Discourse and Understanding. Evidence from English and French. Oxford: Clarendon Press.

Cornish, F. 2008. How Indexicals Function in Texts: Discourse, Text and One Neo-Gricean Account of Indexical Reference. Journal of Pragmatics 40 (6) : 997-ıoı8.

Cornish, F. 2009a. Text and Discourse as Context: Discourse Anaphora and the FDG Contextual Component. Web Papers in Functional Discourse Grammar (WP-FDG) 82 : 97-II5. En ligne à l'adresse suivante: http://home.hum.uva.nl/fdg/working_papers/ WP-FDG-82_Cornish.pdf.

Cornish, F. 2009b. Inter-sentential Anaphora and Coherence Relations in Discourse: A Perfect Match. Language Sciences 3I (5): 572-592.

Cornish, F. 2009c. Le rôle des anaphores dans la mise en place des relations de cohérence: l'hypothèse de J. R. Hobbs. Journal of French Language Studies I9 (2) : 159-I8I.

Cornish, F. 2oroa. Indexicaux, discours, et mémoire discursive. Ce que les premiers révèlent du second et de la troisième. $\operatorname{Linx} 62-63:$ III-I33.

Cornish, F. 2oiob. Anaphora: Text-Based or Discourse-Dependent? Functionalist vs. Formalist Accounts. Functions of Language I7 (2): 207-24I.

Cornish, F. 20II. «Strict» Anadeixis, Discourse Deixis and Text Structuring. Language Sciences $33(5): 753-767$.

De Mulder, W. et Carlier, A. 2006. Du démonstratif à l'article défini: le cas de ce en français moderne. Langue française $\mathrm{I}_{2} 2$ (4) : 96-II3. 
De Mulder, W. et Vetters, K. 2008. Le sens fondamental de maintenant: un token-reflexive. In M. Vuillaume (éd.), Ici et maintenant. Cahiers Chronos 20. Amsterdam - New York: Rodopi : $15-33$.

Diessel, H. 1999. Demonstratives. Form, Function, and Grammaticalization. Amsterdam Philadelphie: J. Benjamins.

Dowty, D. R. 1986. The Effects of Aspectual Class on the Temporal Structure of Discourse: Semantics or Pragmatics? Linguistics and Philosophy 9: 37-6I.

Ehlich, K. 1982. Anaphora and Deixis: Same, Similar, or Different? In R. J. Jarvella et W. KleIn (éd.), Speech, Place and Action. Studies in Deixis and Related Topics. ChichesterNew York: J. Wiley: 315-338.

Fox, B. A. 1987. Discourse Structure and Anaphora. Written and Conversational English. Cambridge - New York - Melbourne : Cambridge University Press.

FrICKE, E. 2003. Origo, Pointing, and Conceptualisation: What Gestures Reveal about the Nature of the Origo in Face-to-Face Interaction. In F. Lenz (éd.), Deictic Conceptualisation of Space, Time and Person. Amsterdam - Philadelphie: J. Benjamins: 69-93.

Gerner, M. 2009. Deictic Features of Demonstratives: A Typological Survey with Special Reference to the Miao Group. Canadian Journal of Linguistics/Revue canadienne de linguistique 54 (I) : 43-90.

Grice, H. P. 1975. Logic and Conversation. In P. Cole et J. Morgan (éd.), Syntax and Semantics. New York - Londres: Academic Press. Vol. 3: Speech Acts: 4I-58.

Gundel, J. K., Hedberg, N. et Zacharski, R. 1993. Cognitive Status and the Form of Referring Expressions in Discourse. Language 69 (2): 274-307.

Hausendorf, H. 2003. Deixis and Speech Situation Revisited. The Mechanism of Perceived Perception. In F. Lenz (éd.), Deictic Conceptualisation of Space, Time and Person. Amsterdam - Philadelphie: J. Benjamins: 249-268.

Kehler, A. 2008. Rethinking the SMASH Approach to Pronoun Interpretation. In J. K. Gundel et N. Hedberg (éd.), Reference. Interdisciplinary Perspectives. Oxford - New York: Oxford University Press: 95-122.

Kehler, A., Kertz, L., Rohde, H. et Elman, J. L. 2008. Coherence and Coreference Revisited. Journal of Semantics 25 (I): I-44.

KibriK, A. A. 20II. Reference in Discourse. Oxford - New York: Oxford University Press.

Kleiber, G. 2008. Comment fonctionne ICI? In M. Vuillaume (éd.), Ici et maintenant. Cahiers Chronos 20. Amsterdam - New York: Rodopi : II3-I45.

Langacker, R. W. 2002a. Deixis and Subjectivity. In F. Brisard (éd.), Grounding. The Epistemic Footing of Deixis and Reference. Berlin - New York: Mouton de Gruyter: I-27.

LANGACKER, R. W. 2002b. Remarks on the English Grounding Systems. In F. BRISARD (éd.), Grounding. The Epistemic Footing of Deixis and Reference. Berlin - New York: Mouton de Gruyter: 29-38.

Lyons, J. 1975. Deixis as the Source of Reference. In E. L. KeEnAn (éd.), Formal Semantics of Natural Language. Cambridge - New York - Melbourne: Cambridge University Press: 6I-83. 
McCarthy, M. 1994. It, This and That. In M. Coulthard (éd.), Advances in Written Text Analysis. London - New York: Routledge: 266-275.

Prévot, L., VIEU, L. et Asher, N. 2009. Une formalisation plus précise pour une annotation moins confuse: la relation d'Élaboration d'entité. Journal of French Language Studies I9 (2): 207-228.

Prince, E. F. 198I. On the Inferencing of Indefinite This NPs. In A. K. Joshi, B. L. Webber et I. A. SAG (éd.), Elements of Discourse Understanding. Cambridge - London - New York: Cambridge University Press : 23I-250.

Pu, M.-M. 20II. Discourse Anaphora: A Cognitive-Functional Account. Munich : LINCOM Europa.

Rothstein, S. D. 2004. Structuring Events. A Study in the Semantics of Lexical Aspect. Malden: Blackwell.

Sмiтн, C. S. 2003. Modes of Discourse. The Local Structure of Texts. Cambridge: Cambridge University Press.

Stukenbrock, A. 20I4. Pointing to an «Empty» Space: Deixis am Phantasma in Face-to-Face Interaction. Journal of Pragmatics 74: 70-93.

Webber, B. L. 1991. Structure and Ostension in the Interpretation of Discourse Deixis. Language and Cognitive Processes 6 (2): 107-I35.

Zwaan, R. A. et Rapp, D. N. 2006. Discourse Comprehension. In M. J. Traxler et M. A. Gernsbacher (éd.), Handbook of Psycholinguistics. Amsterdam: Academic Press - Elsevier: $725^{-764}$. 